\title{
Investigating the Marketing Channels of Agricultural Crops in West Azerbaijan Province, Iran
}

\author{
Mohammad Jalalzadeh ${ }^{1}$, Seyed Hedayatollah Nouri Zamanabadi ${ }^{1} \&$ Khalil Kalantari $^{2}$ \\ ${ }^{1}$ University of Isfahan, Iran \\ ${ }^{2}$ University of Tehran, Iran \\ Correspondence: Mohammad Jalalzadeh, No. 20, Dehghan Avenue, Alborz Street, Orumiyeh, West Azerbaijan, \\ Iran. E-mail: m.jalalzadeh@iran.ir
}

\author{
Received: January 4, 2014 Accepted: February 10, 2014 Online Published: May 21, 2014 \\ doi:10.5539/ijms.v6n3p148 URL: http://dx.doi.org/10.5539/ijms.v6n3p148
}

\begin{abstract}
Marketing is an amazing science nowadays. Marketing methods especially in third world countries must be developed until can Compete with other strong countries to achieve food security and food safety. Based on the statistics, 31.54 percent of the Iran total population lives in rural areas where agriculture is the main source of their income. One of the major problems of agricultural economy in Iran refers to many brokers and intermediaries in the transfer of goods from the producer to the consumer. This reduces the producer's share of the proceeds from the sale of products. Selecting an appropriate marketing channel is effective to alleviate these negative impacts. So in this research have been tried to know and recognize the best marketing channels of Agricultural Crops in West Azerbaijan Province, Iran. In this regard, 8 main marketing channels were recognized and findings revealed that relation among most applied marketing channel and second job is in the highest and with the gender is in the lowest state. Results from Comparison tests of research groups imply that there are significant differences among farmers with different fields of study, different main job, different A Location and different second jobs in applying most applied marketing channel.
\end{abstract}

Keywords: marketing channels, agricultural crops, West Azerbaijan, Iran

\section{Introduction}

West Azerbaijan province produces about 30 percent of total apple production in Iran and is allocated to the first position (Statistical Yearbook of Agriculture, 2004). However, despite of having 7 frontier areas and 9 active customs, the main market is affected by the brokers and intermediaries. Based on available statistics, west Azerbaijan with about 13 million dollars exports has the 4th rank of agricultural exporters in Iran. Approximately 16 million dollars of agricultural commodities produced in the West Azerbaijan province have not been exported from formal customs and areas in fact only 42 percent of agricultural export commodity has been exported from the customs Province (Iran exporting Workgroup, 2011). Jalalzadeh (2008), in his research confirmed that more than 60 percent of Apple growers in West Azerbaijan sell their products through intermediaries. The results of this research can draw an approach for related organizations to design and implement suitable and effective strategies enhancing benefits for both producers and consumers and finally help to sustainable rural and agricultural development.

\section{Literature Review}

\subsection{Agricultural Marketing}

The new concept of marketing was considered since 1960 and the focus shifted from the product to the customer In the past, persuade the potential customers to purchase the product was the Instrument to achieve greater profitability, but in new Paradigm, all elements of the marketing mix (4P) are constituted the instruments to achieve this goal. These elements are product, price, persuade, channel and location of distribution (Keegan, 2001). Boluriyan Tehrani (2001), believed that the elements of marketing mix are: Product, Place Distribution, Price, Packaging, Public Relations, People, Power and Promotion. Agricultural marketing is a form of marketing that encompasses all goods and services related to the field of agriculture. All these products directly or indirectly support the effort to produce and deliver agricultural products from the farm to the consumer (wise GEEK, 2013). Sedaghat (2000), in his research about the problems of pistachio marketing recognized that the 
pistachio marketing is ineffective. Ashrafi et al. (2005) revealed that the share of intermediaries is very high in agricultural trades while Mehdipour et al. (2005), emphasizes on high rate of agricultural marketing margin in Iran. Rinarts et al. (2005) and Dong (2007), believed that Lack of effective communication between the producer and the consumer can enhance rate of agricultural marketing margin. Mutual effective relations between the producer and the consumer have mentioned in Mizuno et al. (2008) and B. Jama (2008) researches too.

\subsection{Agricultural Marketing Channels}

Roosta et al. (2009), in their research believes that major marketing channels of agricultural crops are:

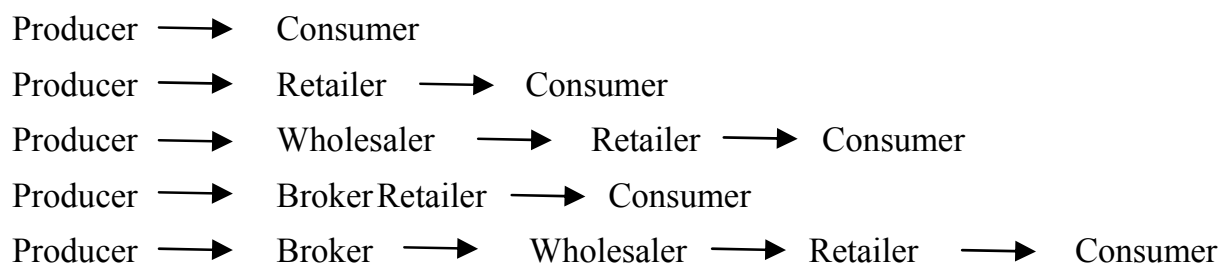

Badsar (2002) says that brokers are Undeniable subject but main problem is the Proportion between the role and share of brokers in marketing process. Results of Salem (2000) showed that 8 main marketing channels are current in the Pomegranate marketing and 4 routes leads to exporting and the others are related to domestic consumer. Also the less marketing margin is in which rout that producer deliver his production to retailer directly and the most marketing margin is related to when the producer deliver his production to wholesaler. Moreover the most share of producer is while producer deliver his crop to retailer. Azizi (2006) has discovered five major marketing channels for rice marketing in Gilan province and states that farmers are forecasting future prices by attention to last month's prices changes trend, and so based on the results of this act, marketing channels and the time to supply of good is determined.

\section{Methodology}

The study used a survey design for data collection. All farmers of West Azerbaijan province were included in the study. The sample size was determined as equal to 386 people through Cochran formula and the stratified proportionate random sampling method was used to choose the sample and a questionnaire was designed and employed to gather the required data. Cronbach's alpha computed to measure reliability of the questionnaire and its rate was 0.86 . Face validity of the instrument was determined by related experts. Data was analyzed using descriptive and inferential statistics such as: percentage, frequency, T-Test, Mann-Whitney (U-Test), Eta test and One-way ANOVA.

\subsection{Study Area}

West Azerbaijan produces about 30 percent of total apple production in Iran and the first place in this regard is allocated to this province. Despite having several customs and markets with possibility of direct deal between producer and customer, major share of agricultural trade is for big intermediaries. Based on statistics, West Azerbaijan with revenue about 13 million dollars ( 0.59 percent than total of country) from agricultural exports has a very important problem in which only 42 percent of total province exports has done from formal borders and gates and the remaining have been exported from unknown gates (country export workgroup, 2011). Jalalzadeh (2008) believed that about 60 percent of apple farmers in study area use than marketing channels which are under the intermediaries.

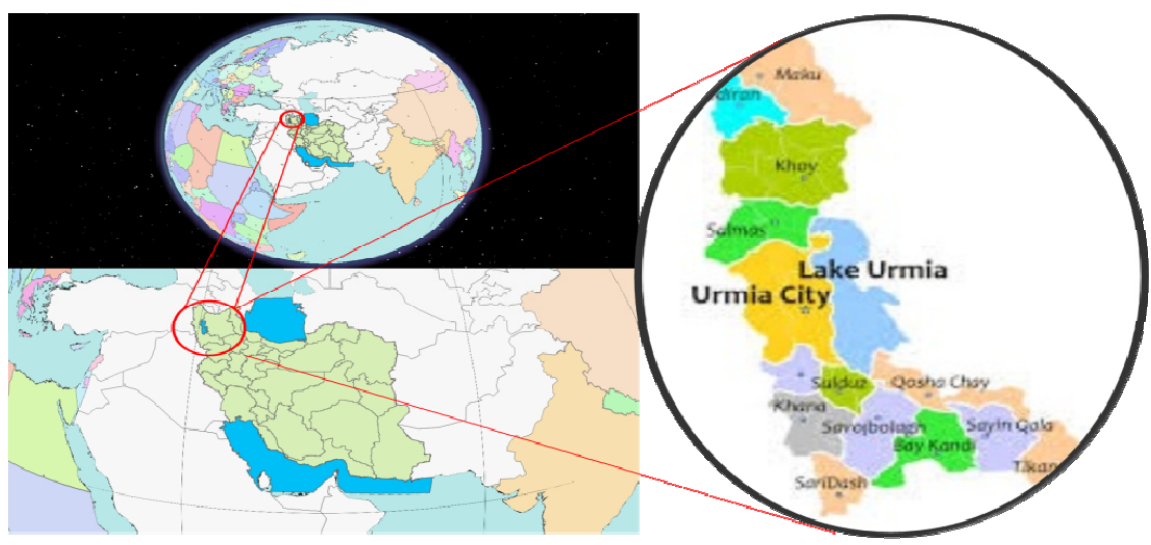

Figure 1. Geographical position of West Azerbaijan 


\section{Results and Discussion}

\subsection{Professional and Individual Characteristics of the Farmers}

In total, 366 persons ( 94.8 percent) of the grape farmers are men and there are merely 20 (5.2 percent) female farmers. The average age of the farmers is 49 years and their average farming experience of the respondents is 24 years. Considering the educational level, most of the farmers $(57.5$ percent) have reading \& writing skill. According to the results of this research, the main job of 292 of the respondents (75.6 percent) is agriculture and the others have non-agricultural as their main jobs and farming is a part-time work for them (Table1).

Table 1. The individual and professional characteristics of the farmers of West Azerbaijan province, 2013 ( $n=386$ )

\begin{tabular}{|c|c|c|c|c|c|c|}
\hline & & Average & \multicolumn{2}{|c|}{ Minimum } & Maximum & $\begin{array}{l}\text { Standard } \\
\text { deviation }\end{array}$ \\
\hline Age & & 49 & 78 & & 23 & 12.318 \\
\hline \multirow[t]{2}{*}{ Farming experience } & & 24 & 1 & & 59 & 14.190 \\
\hline & & \multicolumn{2}{|c|}{ Frequency } & Percentage & \multicolumn{2}{|c|}{ Cumulative percentage } \\
\hline \multirow[t]{2}{*}{ - $\quad$ Gender } & Male & 366 & & 94.8 & \multicolumn{2}{|l|}{94.8} \\
\hline & Female & 20 & & 5.2 & 100 & \\
\hline \multirow[t]{4}{*}{ - $\quad$ Educational level } & Illiterate & 45 & & 11.7 & \multicolumn{2}{|l|}{11.7} \\
\hline & reading \& writing skill & 222 & & 57.5 & 69.2 & \\
\hline & Diploma & 75 & & 19.4 & 88.6 & \\
\hline & Post- diploma & 44 & & 11.4 & 100 & \\
\hline \multirow[t]{2}{*}{ - $\quad$ Main job } & Agriculture & 292 & & 75.6 & 75.6 & \\
\hline & Non-Agriculture & 94 & & 24.4 & 100 & \\
\hline
\end{tabular}

Source: Research results.

\subsection{Status of Agricultural Crops Consumption}

Results showed that farmers are consuming their products in four ways which are respectively:

1) Selling to Markets

2) Selling to Relatives

3) Individual Consumption

4) Giving to Relatives

\subsection{Marketing Channels}

Based on the findings, 8 major marketing channels were recognized that are:

1) Producer $\rightarrow$ Consumer

2) Producer $\rightarrow$ Wholesaler $\rightarrow$ Retailer $\rightarrow$ Consumer

3) Producer $\rightarrow$ Broker $\rightarrow$ Wholesaler $\rightarrow$ Retailer $\rightarrow$ Consumer

4) Producer $\rightarrow$ Broker $\rightarrow$ Retailer $\rightarrow$ Consumer

5) Producer $\rightarrow$ Retailer $\rightarrow$ Consumer

6) Sales to broker on harvesting time

7) Producer $\rightarrow$ Food

8) Sales to broker before harvesting time

\subsection{Applying Marketing Channels}

In this section farmers asked to give from 0 to 100 percent to marketing channels given to their share of applying. Results showed that, channels (2) and (3) are respectively the most and less applied channels by the farmers (Table 2). 
Table 2. Distribution of respondents based on the applying marketing channels, $2013(n=386)$

\begin{tabular}{|c|c|c|c|c|c|c|c|}
\hline Channel(1) & Frequency & Percentage & $\begin{array}{l}\text { Cumulative } \\
\text { Percentage }\end{array}$ & Channel(5) & Frequency & Percentage & $\begin{array}{l}\text { Cumulative } \\
\text { Percentage }\end{array}$ \\
\hline Less than 5 & 187 & 48.4 & 48.4 & $0-1$ & 292 & 75.6 & 75.6 \\
\hline $5-10$ & 66 & 17.1 & 65.6 & $1-2$ & 93 & 24.1 & 99.7 \\
\hline $10-20$ & 52 & 13.5 & 79 & More than 2 & 1 & 0.3 & 100 \\
\hline $20-30$ & 67 & 17.4 & 96.4 & Total & 386 & 100 & \\
\hline More than 30 & 14 & 3.6 & 100 & & & & \\
\hline Total & 386 & 100 & & & & & \\
\hline Max: 32 & Mode: 0 & Median: 6 & Avarage: 9.14 & Max: 4 & Mode: 0 & Median: 1 & Avarage: 0.81 \\
\hline $\begin{array}{l}\text { S.Erroe: } \\
0.504\end{array}$ & $\begin{array}{l}\text { S.Deviation: } \\
9.92\end{array}$ & $\begin{array}{l}\text { Variation: } \\
98.38\end{array}$ & Min: 0 & $\begin{array}{l}\text { S.Erroe: } \\
0.041\end{array}$ & $\begin{array}{l}\text { S.Deviation: } \\
0.82\end{array}$ & $\begin{array}{l}\text { Variation: } \\
0.67\end{array}$ & Min: 0 \\
\hline Channel(2) & Frequency & Percentage & $\begin{array}{l}\text { Cumulative } \\
\text { Percentage }\end{array}$ & Channel(6) & Frequency & Percentage & $\begin{array}{l}\text { Cumulative } \\
\text { Percentage }\end{array}$ \\
\hline $10-30$ & 26 & 6.7 & 6.7 & Less than 2 & 263 & 68.1 & 68.1 \\
\hline $30-50$ & 128 & 33.2 & 39.9 & $2-4$ & 99 & 25.6 & 93.7 \\
\hline $50-70$ & 82 & 21.2 & 61.1 & $4-6$ & 23 & 6 & 99.7 \\
\hline $70-90$ & 11 & 2.8 & 64 & More than 6 & 1 & 0.3 & 100 \\
\hline More than 90 & 139 & 36 & 100 & Total & 386 & 100 & \\
\hline Total & 386 & 100 & & & & & \\
\hline Max: 100 & Mode: 100 & Median: 55 & Avarage: 66 & Max: 7 & Mode: 0 & Median: 2 & Avarage: 1.7 \\
\hline $\begin{array}{l}\text { S.Erroe: } \\
0.67\end{array}$ & $\begin{array}{l}\text { S.Deviation: } \\
1.400\end{array}$ & $\begin{array}{l}\text { Variation: } \\
756.75\end{array}$ & Min: 11 & $\begin{array}{l}\text { S.Erroe: } \\
1.735\end{array}$ & $\begin{array}{l}\text { S.Deviation: } \\
1.65\end{array}$ & $\begin{array}{l}\text { Variation: } \\
2.73\end{array}$ & Min: 0 \\
\hline Channel(3) & Frequency & Percentage & $\begin{array}{l}\text { Cumulative } \\
\text { Percentage }\end{array}$ & Channel(7) & Frequency & Percentage & $\begin{array}{l}\text { Cumulative } \\
\text { Percentage }\end{array}$ \\
\hline $0-1$ & 382 & 99 & 99 & Less than 10 & 248 & 64.2 & 64.2 \\
\hline $1-2$ & 3 & 0.7 & 99.7 & $10-30$ & 118 & 30.6 & 94.8 \\
\hline More than 2 & 1 & 0.3 & 100 & $30-50$ & 19 & 4.9 & 99.7 \\
\hline Total & 386 & 100 & & $\begin{array}{l}\text { More than } 50 \\
\text { Total }\end{array}$ & $\begin{array}{l}1 \\
386\end{array}$ & $\begin{array}{l}0.3 \\
100\end{array}$ & 100 \\
\hline $\begin{array}{l}\text { Max: } 3 \\
\text { S.Erroe: } \\
0.012\end{array}$ & $\begin{array}{l}\text { Mode: } 0 \\
\text { S.Deviation: } \\
0.253\end{array}$ & $\begin{array}{l}\text { Median: } 0 \\
\text { Variation: } \\
0.064\end{array}$ & $\begin{array}{l}\text { Avarage: } 0.03 \\
\text { Min: } 0\end{array}$ & $\begin{array}{l}\text { Max: } 65 \\
\text { S.Erroe: } \\
0.540\end{array}$ & $\begin{array}{l}\text { Mode: } 0 \\
\text { S.Deviation: } \\
10.62\end{array}$ & $\begin{array}{l}\text { Median: } 5.5 \\
\text { Variation: } \\
112.81 \\
\end{array}$ & $\begin{array}{l}\text { Avarage: } 9.3 \\
\text { Min: } 0\end{array}$ \\
\hline Channel(4) & Frequency & Percentage & $\begin{array}{l}\text { Cumulative } \\
\text { Percentage }\end{array}$ & Channel(8) & Frequency & Percentage & $\begin{array}{l}\text { Cumulative } \\
\text { Percentage }\end{array}$ \\
\hline Less than 2 & 251 & 65 & 65 & Less than 5 & 167 & 43.3 & 43.3 \\
\hline $2-4$ & 134 & 34.7 & 99.7 & $5-10$ & 43 & 11.1 & 54.4 \\
\hline $4-6$ & 0 & 0 & 99.7 & $10-20$ & 61 & 15.8 & 70.2 \\
\hline More than 8 & 1 & 0.3 & 100 & $20-30$ & 101 & 26.2 & 96.4 \\
\hline Total & 386 & 100 & & $\begin{array}{l}\text { More than } 30 \\
\text { Total }\end{array}$ & $\begin{array}{l}14 \\
386\end{array}$ & $\begin{array}{l}3.6 \\
100\end{array}$ & 100 \\
\hline Max: 7 & Mode: 0 & Median: 2 & Avarage: 1.6 & Max: 32 & Mode: 0 & Median: 10 & Avarage: 11.3 \\
\hline $\begin{array}{l}\text { S.Erroe: } \\
2.04\end{array}$ & $\begin{array}{l}\text { S.Deviation: } \\
1.43\end{array}$ & $\begin{array}{l}\text { Variation: } \\
0.072\end{array}$ & Min: 1 & $\begin{array}{l}\text { S.Erroe: } \\
0.572\end{array}$ & $\begin{array}{l}\text { S.Deviation: } \\
11.23\end{array}$ & $\begin{array}{l}\text { Variation: } \\
126.11\end{array}$ & Min: 0 \\
\hline
\end{tabular}

Source: Research results.

\subsection{Favorite Marketing Channels}

In this section farmers asked to give from 0 to 10 points to favorite marketing channels. Based on the findings, channel (1) is the most favorable channel and the channel (4) has the lowest rate from viewpoints of farmers (Table $3)$. 
Table 3. Distribution of respondents based on the favorite marketing channels, 2013( $n=386$ )

\begin{tabular}{|c|c|c|c|c|c|c|c|}
\hline Channel(1) & Frequency & Percentage & $\begin{array}{l}\text { Cumulative } \\
\text { Percentage } \\
\end{array}$ & Channel(5) & Frequency & Percentage & $\begin{array}{l}\text { Cumulative } \\
\text { Percentage }\end{array}$ \\
\hline $0-2$ & 0 & 0 & 0 & $0-2$ & 1 & 0.3 & 0.3 \\
\hline $2-4$ & 0 & 0 & 0 & $2-4$ & 1 & 0.3 & 0.6 \\
\hline $4-6$ & 7 & 1.8 & 1.8 & $4-6$ & 8 & 2.1 & 2.7 \\
\hline $6-8$ & 108 & 28 & 29.8 & $6-8$ & 122 & 31.6 & 34.3 \\
\hline More than 8 & 271 & 70.2 & 100 & More than 8 & 254 & 65.7 & 100 \\
\hline Total & 386 & 100 & & Total & 386 & 100 & \\
\hline Max: 9 & Mode: 9 & Median: 9 & Avarage: 8.6 & Max: 10 & Mode: 9 & Median: 9 & Avarage: 8.5 \\
\hline $\begin{array}{l}\text { S.Erroe: } \\
0.036\end{array}$ & $\begin{array}{l}\text { S.Deviation: } \\
0.711\end{array}$ & $\begin{array}{l}\text { Variation: } \\
0.506\end{array}$ & Min: 5 & $\begin{array}{l}\text { S.Erroe: } \\
0.045\end{array}$ & $\begin{array}{l}\text { S.Deviation: } \\
0.93\end{array}$ & $\begin{array}{l}\text { Variation: } \\
0.82\end{array}$ & Min: 0 \\
\hline Channel(2) & Frequency & Percentage & $\begin{array}{l}\text { Cumulative } \\
\text { Percentage }\end{array}$ & Channel(6) & Frequency & Percentage & $\begin{array}{l}\text { Cumulative } \\
\text { Percentage } \\
\end{array}$ \\
\hline $0-2$ & 0 & 0 & 0 & $0-2$ & 269 & 69.7 & 69.7 \\
\hline $2-4$ & 0 & 0 & 0 & $2-4$ & 70 & 18.1 & 87.8 \\
\hline $4-6$ & 91 & 23.6 & 23.6 & $4-6$ & 46 & 11.9 & 99.7 \\
\hline $6-8$ & 108 & 28 & 51.6 & $6-8$ & 1 & 0.3 & 100 \\
\hline More than 8 & 187 & 48.4 & 100 & More than 8 & 0 & 0 & 0 \\
\hline Total & 386 & 100 & & Total & 386 & 100 & \\
\hline Max: 9 & Mode: 10 & Median: 8 & Avarage: 8.32 & Max: 7 & Mode: 0 & Median: 1 & Avarage: 1.5 \\
\hline $\begin{array}{l}\text { S.Erroe: } \\
0.087\end{array}$ & $\begin{array}{l}\text { S.Deviation: } \\
1.716\end{array}$ & $\begin{array}{l}\text { Variation: } \\
2.94\end{array}$ & Min: 5 & $\begin{array}{l}\text { S.Erroe: } \\
0.088\end{array}$ & $\begin{array}{l}\text { S.Deviation: } \\
1.74\end{array}$ & $\begin{array}{l}\text { Variation: } \\
3.03\end{array}$ & Min: 0 \\
\hline Channel(3) & Frequency & Percentage & $\begin{array}{l}\text { Cumulative } \\
\text { Percentage }\end{array}$ & Channel(7) & Frequency & Percentage & $\begin{array}{l}\text { Cumulative } \\
\text { Percentage } \\
\end{array}$ \\
\hline $0-2$ & 282 & 73.1 & 73.1 & $0-2$ & 331 & 85.8 & 85.8 \\
\hline $2-4$ & 57 & 14.7 & 87.8 & $2-4$ & 54 & 14 & 99.7 \\
\hline $4-6$ & 47 & 12.2 & 100 & $4-6$ & 1 & 0.3 & 100 \\
\hline $6-8$ & 0 & 0 & 0 & $6-8$ & 0 & 0 & 0 \\
\hline More than 8 & 0 & 0 & 0 & More than 8 & 0 & 0 & 0 \\
\hline Total & 386 & 100 & & Total & 386 & 100 & \\
\hline Max: 9 & Mode: 10 & Median: 8 & Avarage: 8.32 & Max: 6 & Mode: 0 & Median: 0 & Avarage: 0.7 \\
\hline $\begin{array}{l}\text { S.Erroe: } \\
0.087 \\
\end{array}$ & $\begin{array}{l}\text { S.Deviation: } \\
1.716 \\
\end{array}$ & $\begin{array}{l}\text { Variation: } \\
2.94\end{array}$ & Min: 5 & $\begin{array}{l}\text { S.Erroe: } \\
0.056 \\
\end{array}$ & $\begin{array}{l}\text { S.Deviation: } \\
1.10 \\
\end{array}$ & $\begin{array}{l}\text { Variation: } \\
1.23\end{array}$ & Min: 0 \\
\hline Channel(4) & Frequency & Percentage & $\begin{array}{l}\text { Cumulative } \\
\text { Percentage }\end{array}$ & Channel(8) & Frequency & Percentage & $\begin{array}{l}\text { Cumulative } \\
\text { Percentage } \\
\end{array}$ \\
\hline $0-2$ & 367 & 95.1 & 95.1 & $0-2$ & 356 & 92.2 & 92.2 \\
\hline $2-4$ & 14 & 3.6 & 98.7 & $2-4$ & 25 & 6.5 & 98.7 \\
\hline $4-6$ & 5 & 1.3 & 100 & $4-6$ & 5 & 1.3 & 100 \\
\hline $6-8$ & 0 & 0 & 0 & $6-8$ & 0 & 0 & 0 \\
\hline More than 8 & 0 & 0 & 0 & More than 8 & 0 & 0 & 0 \\
\hline Total & 386 & 100 & & Total & 386 & 100 & \\
\hline Max: 6 & Mode: 0 & Median: 0 & Avarage: 0.66 & Max:6 & Mode: 0 & Median: 0 & Avarage: 0.67 \\
\hline $\begin{array}{l}\text { S.Erroe: } \\
0.047\end{array}$ & $\begin{array}{l}\text { S.Deviation: } \\
0.934\end{array}$ & $\begin{array}{l}\text { Variation: } \\
0.87\end{array}$ & Min: 0 & $\begin{array}{l}\text { S.Erroe: } \\
0.051\end{array}$ & $\begin{array}{l}\text { S.Deviation: } \\
1.003\end{array}$ & $\begin{array}{l}\text { Variation: } \\
1.006\end{array}$ & Min: 0 \\
\hline
\end{tabular}

Source: Research results.

\subsection{Relation among Most Applied Marketing Channel and Variables}

Eta-test findings showed that Relation among most applied marketing channel (channel 2) and second job is in the highest and with the gender is in the lowest state (Table 4). 
Table 4. Relation among most applied marketing channel and criterion variables, 2013( $\mathrm{n}=386)$

\begin{tabular}{lll}
\hline Variable1 & Variable1 & Eta \\
\hline & Second job & 0.592 \\
& Field of Study & 0.565 \\
Applying Most Applied Marketing Channel & A Location & 0.501 \\
& Main job & 0.490 \\
& gender & 0.357 \\
\hline
\end{tabular}

Source: Research results.

\subsection{Comparison of Research Groups}

The results of U-Test imply that there is significant difference between farmers have agricultural related fields of study and unrelated in applying most applied marketing channel (table 5).

According to the resultant data of $\mathrm{t}$-Test (table 6), there is significant difference between farmers with different main job and A Location in applying most applied marketing channel.

Also based on the findings of F-Test, groups of farmers with different second jobs are different in applying most applied marketing channel (table 7).

Table 5. Comparison of research groups by U-test

\begin{tabular}{llllll}
\hline Main variable & Grouping variable & Groups & $\begin{array}{l}\text { Rank } \\
\text { Average }\end{array}$ & Z & Sig \\
\hline $\begin{array}{lllll}\text { Applying Most Applied } \\
\text { Marketing Channel }\end{array}$ & Field of study & $\begin{array}{l}\text { Related with } \\
\text { agriculture }\end{array}$ & 111 & $5.165^{* * *}$ & 0.000 \\
\cline { 2 - 3 } & & $\begin{array}{l}\text { Unrelated with } \\
\text { agriculture }\end{array}$ & 54.81 & \\
\hline
\end{tabular}

Note. $* *$ Significance at the level of 1 percent.

Table 6. Comparison of research groups by t-test

\begin{tabular}{|c|c|c|c|c|c|}
\hline Main variable & Grouping variable & Groups & Average & $\mathbf{t}$ & Sig \\
\hline \multirow{2}{*}{$\begin{array}{l}\text { Applying Most Applied } \\
\text { Marketing Channel }\end{array}$} & Main job & $\begin{array}{l}\text { Agriculture } \\
\text { Non-agriculture }\end{array}$ & $\begin{array}{l}68.93 \\
56.88\end{array}$ & $4.11^{* *}$ & 0.000 \\
\hline & A Location & $\begin{array}{l}\text { City } \\
\text { Village }\end{array}$ & $\begin{array}{l}59.53 \\
66.50\end{array}$ & $-1.62^{* * *}$ & 0.000 \\
\hline
\end{tabular}

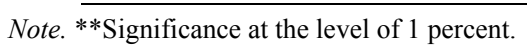

Table 7. Comparison of research groups by F-test (One-way ANOVA)

\begin{tabular}{|c|c|c|c|c|c|c|c|c|}
\hline \multirow[t]{2}{*}{ Main variable } & \multirow[t]{2}{*}{ Grouping variable } & \multirow[t]{2}{*}{ Groups } & \multirow{2}{*}{$\begin{array}{l}\text { Rank } \\
\text { Average }\end{array}$} & \multirow[t]{2}{*}{$\mathbf{F}$} & \multirow[t]{2}{*}{ Sig } & \multicolumn{3}{|l|}{ LSD } \\
\hline & & & & & & $1 \& 2$ & $1 \& 3$ & $2 \& 3$ \\
\hline \multirow{3}{*}{$\begin{array}{l}\text { Applying Most Applied } \\
\text { Marketing Channel }\end{array}$} & \multirow{3}{*}{ Main job } & Without second job & 55.28 & \multirow{3}{*}{$43.26^{* *}$} & \multirow{3}{*}{0.000} & \multirow{3}{*}{$*$} & \multirow{3}{*}{-} & \multirow{3}{*}{$*$} \\
\hline & & $\begin{array}{l}\text { Agricultural related } \\
\text { second job }\end{array}$ & 77.04 & & & & & \\
\hline & & $\begin{array}{l}\text { Un-agricultural } \\
\text { related second job }\end{array}$ & 52.33 & & & & & \\
\hline
\end{tabular}

\section{Acknowledgements}

The authors gratefully acknowledge the Department of rural planning, University of Isfahan, Iran for equipment support. The authors also thank of Agricultural extension organization experts of West Azerbaijan province for cooperation with authors. 


\section{References}

Ashrafi, M., Sadrolashrafi, M., \& Karbasi, A. (2005). Investigating the marketing margin of grapes and raisins in Iran. Journal of Commercial Bulletin, 35, 213-238.

Azizi, J. (2006). Economic appraisal of rice marketing strategies in Gilan province. Journal of agricultural science. Azad University.

Badsar, M. (2002). Investigating the ideas of agricultural crops marketing in member farmers of rural cooperatives (Bookan County). Master Thesis in Rural development, Tehran University, University College of Agriculture \& Natural Resources.

Boluriyan, T. M. (2001). Marketing and market management. Publication of institute of commercial studies and researches. Tehran, Iran.

Dong, W., Swain, S., \& Berger, P. (2007). The role of channel quality in customer equity management. Journal of Business Research, 60. http://dx.doi.org/10.1016/j.jbusres.2007.05.005

Export workgroup of Iran. (2011). Monitoring the provincial foreign trade. Publication of Trade Promotion Organization of Iran, Ministry of Industry, Mine and Trade.

Geravand, A., Noorayi, M., \& Saei, I. (2010). The impact of marketing mix on purchasing decisions and customer satisfaction in production cooperatives of Kouhdasht county. Journal of Cooperation.

Jalalzadeh, M. (2008). Investigation and Analyzing the Components of Improvement of Gardens Management in Orumieh County. Master Thesis in Rural Development, Tehran University, Karaj.

Jama, B., Mohamed, A., Mulatya, J., \& Njui, A. (2008). Comparing the "Big Five": A framework for the sustainable management of indigenous fruit trees in the dry lands of East and Central Africa. Ecological Indicators. http://dx.doi.org/10.1016/j.ecolind.2006.11.009

Keegan, W. (1936). Management of global marketing. Publication of cultural research office, Tehran, Iran.

Mehdipoor, E., Sadrolashrafi, M., \& Karbasi, A. (2005). Investigating the Marketing of Potato Product in Iran. Journal of Agricultural Science.

Mizuno, M., Saji, A., Sumita, U., \& Suzuki, H. (2008). Optimal threshold analysis of segmentation methods for identifying target customers. European Journal of Operational Research. http://dx.doi.org/10.1016/j.ejor.2007.01.038

Reinartz, W., Thomas, J., \& Kumar, V. (2005). Balancing acquisition and retention resources to maximize customer profitability. $J$ Mark, 69(1). http://dx.doi.org/10.1509/jmkg.69.1.63.55511

Roosta, A., Venus, D., \& Ebrahimi, A. H. (2009). Marketing management. Samt Publication, Tehran, Iran.

Salem, J. (2000). Investigating the problems of pomegranate marketing in Yazd province. Journal of Agricultural Economy and Development.

Sedaghat, R. (2000). Economic evaluation of pistachio marketing in Fars province. Journal of Agricultural Economy and Development, 32, 273-290.

Statistical center of Iran. (2004). Annual agricultural yearbook. Retrieved from http://www.amar.sci.org.ir

Wise Geek. (2013). Retrieved from http://www.wisegeek.com/what-is-agricultural-marketing.htm

\section{Copyrights}

Copyright for this article is retained by the author(s), with first publication rights granted to the journal.

This is an open-access article distributed under the terms and conditions of the Creative Commons Attribution license (http://creativecommons.org/licenses/by/3.0/). 